\title{
НАИЛУЧШИЕ КУБАТУРНЫЕ ФОРМУЛЫ НА МНОЖЕСТВАХ ПЕРИОДИЧЕСКИХ ФУНКЦИЙ
}

В работе используются следующие обозначения:

$$
\begin{gathered}
\|\varphi(\cdot)\|_{q}=\left[\int_{0}^{1}|\varphi(x)|^{q} d x\right]^{1 / q}, \quad\|f(\cdot, \cdot)\|_{q}=\left[\int_{0}^{1} \int_{0}^{1}|f(x, y)|^{q} d x d y\right]^{1 / q}, \\
f^{(j, l)}(x, y)=\frac{\partial^{j+l}}{\partial x^{j} \partial y^{l}} f(x, y),
\end{gathered}
$$

$B_{k}^{*}(x) \equiv B_{k}(x)$ при $x \in[0,1)$ и $B_{k}^{*}(x+1) \equiv B_{k}^{*}(x)$, где $B_{k}(x)-$ многочлен Бернулли степени $k$. Числа $r, s, m, n \geqslant 1,0 \leqslant \varrho_{i}<r \quad(i=$ $=1, \ldots, m), 0 \leqslant \sigma_{k}<s(k=1, \ldots, n), 1<q<\infty, M, P, Q>0$ считаем заданными, $p=q l(q-1)$.

Через $\widetilde{W}_{q}^{r s} \quad$ обозначим множество всех функций $f(x, y)$, которые имеют при всех вещественных значениях $x$ и $y$ непрерывные производные $f^{(i, j)}(x, y) \quad(i=0, \ldots, r-1 ; j=0, \ldots, s-1)$, кусочно-непрерывные производные $f^{(r, j)}(x, y), f^{(i, s)}(x, y) \quad(j=0, \ldots, s ; i=0, \ldots, r)$ и удовлетворяют условиям

$$
\begin{gathered}
f(x+1, y) \equiv f(x, y+1) \equiv f(x, y), \\
\left\|f^{(r, s)}(\cdot, \cdot)\right\|_{q} \leqslant Q, \quad\left\|\varphi_{f}^{(r)}(\cdot)\right\|_{q} \leqslant M, \quad\left\|\psi_{f}^{(s)}(\cdot)\right\|_{q} \leqslant P,
\end{gathered}
$$

где

$$
\varphi_{f}^{(r)}(\cdot)=\int_{0}^{1} f^{(r, 0)}(\cdot, u) d u, \quad \psi_{f}^{(s)}(\cdot)=\int_{0}^{1} f^{(0, s)}(t, \cdot) d t .
$$

Рассмотрим задачу нахождения наилучшей $\left[{ }^{1-2}\right]$ на множестве $\widetilde{W}_{q}^{r s}$ кубатурной формулы вида

$$
\int_{0}^{1} \int_{0}^{1} f(x, y) d x d y=\sum_{i=1}^{m} \sum_{k=1}^{n} \sum_{j=0}^{\rho_{t}} \sum_{l=0}^{\sigma_{k}} A_{i k j f}(j, l)\left(x_{i}, y_{k}\right)+R_{m n}(f) .
$$

Другими словами, найдем значения узлов $\left(x_{i}, y_{k}\right)$ и весов $A_{i k j l}$ $\left(i=1, \ldots, m ; k=1, \ldots, n ; j=0, \ldots, \varrho_{i} ; l=0, \ldots, \sigma_{k}\right)$, которые доставляют наименьшее значение величине

$$
R_{m n}=\sup _{f \in \tilde{\boldsymbol{W}}_{q}}\left|R_{m n}(f)\right| .
$$


Для решения этой задачи нам потребуются следующие леммы.

Л е м м а 1. Пусть функции $\pi_{j}(x)(j=0, \ldots, N)$ линейно независимы в $L_{p}(0,1)$. Для того чтобы числа $a_{0}, \ldots, a_{N}$ доставляли наименьшее значение величине

$$
I=\int_{0}^{1}\left|\sum_{k=0}^{N} a_{k} \pi_{k}(x)\right|^{p} d x
$$

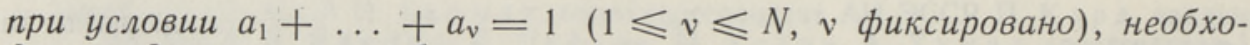
димо и достаточно, чтобы при некотором $\mu$ выполнялись равенства

$$
\int_{0}^{1}\left|\sum_{k=0}^{N} a_{k} \pi_{k}(x)\right|^{p-1} \operatorname{sign}\left(\sum_{k=0}^{N} a_{k} \pi_{k}(x)\right) \pi_{j}(x) d x=\mu_{j} \quad(j=0, \ldots, N),
$$

$\mu_{j}=\mu$ при $1 \leqslant j \leqslant v, \mu_{j}=0$ в остальных случаях, $a_{1}+\ldots+a_{v}=1$.

Д ок а з а ельство. Необходимость условий (3) сразу следует из необходимых условий минимума функции Лагранжа $L=I-$ $-\mu p\left(a_{1}+\ldots+a_{v}-1\right)$.

Докажем достаточность этих условий. Пусть числа $a_{0}, \ldots, a_{N}$ удовлетворяют равенствам (3), $b_{0}, \ldots, b_{N}-$ произвольные числа, $b_{1}+\ldots+b_{v}=1$. Тогда

$$
\int_{0}^{1}\left|\sum_{k=0}^{N} a_{k} \pi_{k}(x)\right|^{p-1} \operatorname{sign}\left(\sum_{k=0}^{N} a_{k} \pi_{k}(x)\right) \sum_{j=0}^{N} b_{j} \pi_{j}(x) d x=\sum_{j=0}^{N} b_{j} \mu_{j}=\mu,
$$

т. е. это выражение не зависит от выбора чисел $b_{0}, \ldots, b_{N}$; в частности,

$$
\int_{0}^{1}\left|\sum_{k=0}^{N} a_{k} \pi_{k}(x)\right|^{p} d x=\int_{0}^{1}\left|\sum_{k=0}^{N} a_{k} \pi_{k}(x)\right|^{p-1} \operatorname{sign}\left(\sum_{k=0}^{N} a_{k} \pi_{k}(x)\right) \sum_{j=0}^{N} b_{j} \pi_{j}(x) d x .
$$

Применяя к правой части этого равенства неравенство Гёльдера, получим неравенство

$$
\int_{0}^{1}\left|\sum_{k=0}^{N} a_{k} \pi_{k}(x)\right|^{p} d x \leqslant \int_{0}^{1}\left|\sum_{k=0}^{N} b_{k} \pi_{k}(x)\right|^{p} d x,
$$

которое и доказывает достаточность условий (3).

Л е м м 2. Пусть $\left\{\pi_{0}(x), \ldots, \pi_{\alpha}(x)\right\} u\left\{\omega_{0}(x), \ldots, \omega_{\beta}(x)\right\}-$ множества линейно независимых в $L_{p}(0,1)$ функций. Для того чтобы числа $a_{i k}(i=1, \ldots, \alpha ; k=1, \ldots, \beta)$, функции $\varphi(x), \psi(y) \in L_{p}(0,1)$ доставляли величине

$$
J=\int_{0}^{1} \int_{0}^{1}\left|\sum_{i=1}^{\alpha} \sum_{k=1}^{\beta} a_{i k} \pi_{i}(x) \omega_{k}(y)+\varphi(x) \omega_{0}(y)+\psi(y) \pi_{0}(x)\right|^{p} d x d y
$$

наименьшее значение при условии

$$
\sum_{i=1}^{v_{1}} \sum_{k=1}^{v_{2}} a_{i k}=1 \quad\left(1 \leqslant v_{1} \leqslant \alpha, 1 \leqslant v_{2} \leqslant \beta, v_{1}, v_{2} \text { фиксированы }\right),
$$

необходимо и достаточно, чтобы при некотором $\lambda$ выполнялись равенства

$$
\begin{aligned}
\int_{0}^{1} \int_{0}^{1}|F(x, y)|^{p-1} \operatorname{sign} F(x, y) \pi_{i}(x) \omega_{k}(y) d x d y=\lambda_{i k} \\
(i=1, \ldots, \alpha ; k=1, \ldots, \beta),
\end{aligned}
$$

$\lambda_{i k}=\lambda$ при $1 \leqslant i \leqslant v_{1}, 1 \leqslant k \leqslant v_{2} ; \lambda_{i k}=0$ в остальных случаях, 
$\sum_{i=1}^{v_{1}} \sum_{k=1}^{v_{2}} a_{i k}=1$

$\int_{0}^{1}|F(x, y)|^{p-1} \operatorname{sign} F(x, y) \pi_{0}(x) d x \equiv 0$,

$\int_{0}^{1}|F(x, y)|^{p-1} \operatorname{sign} F(x, y) \omega_{0}(y) d y \equiv 0$,

гेe

$$
F(x, y)=\sum_{i=1}^{\alpha} \sum_{k=1}^{\beta} a_{i k} \pi_{i}(x) \omega_{k}(y)+\varphi(x) \omega_{0}(y)+\psi(y) \pi_{0}(x) .
$$

Д о к а з а т е л с т в о. Существование наименьшего значения величины (4) при условии (5) следует из относительной слабой компактности ограниченных множеств в $L_{p}(0,1)$ при $1<p<\infty$ и выпуклости величины (4) как функционала от $\varphi(x)$ и $\psi(y)$. Необходимость условий леммы вытекает из необходимых условий минимума функционала Лагранжа $H=J-\lambda p\left(\sum_{i=1}^{v_{1}} \sum_{k=1}^{v_{2}} a_{i k}-1\right)$ и необходимых условий минимума задачи вариационного исчисления.

Докажем достаточность условий леммы. Пусть функция $F(x, y)$ удовлетворяет условиям (6),

$$
F_{1}(x, y)=\sum_{i=1}^{\alpha} \sum_{k=1}^{\beta} b_{i k} \pi_{i}(x) \omega_{k}(y)+\varphi_{1}(x) \omega_{0}(y)+\psi_{1}(y) \pi_{0}(x),
$$

где $b_{i k}(i=1, \ldots, \alpha ; k=1, \ldots, \beta)$ - произвольные числа, $\sum_{i=1}^{v_{1}} \sum_{k=1}^{v_{2}} b_{i k}=1, \varphi_{1}(x), \psi_{1}(y)$ - произвольные функции из $L_{p}(0,1)$. Тогда, в силу (6),

$$
\int_{0}^{1} \int_{0}^{1}|F(x, y)|^{p-1} \operatorname{sign} F(x, y) F_{1}(x, y) d x d y=\lambda,
$$

откуда, как и при доказательстве леммы 1 , получаем достаточность условий (6).

Л м м а 3. Пусть выполнено предположение леммы 2, а числа $b_{0}^{*}, \ldots, b_{\alpha}^{*} ; c_{0}^{*}, \ldots, c_{\beta}^{*}$ доставляют величинам

$$
\int_{0}^{1}\left|\sum_{i=0}^{\alpha} b_{i} \pi_{i}(x)\right|^{p} d x \quad u \quad \int_{0}^{1}\left|\sum_{k=0}^{\beta} c_{k} \omega_{k}(x)\right|^{p} d x
$$

при условиях $b_{1}+\ldots+b_{v_{1}}=1, c_{1}+\ldots+c_{v_{2}}=1$ наименьшие значения, равные соответственно $\gamma_{1} u \gamma_{2}$. Тогда величина (4) при условии (5) достигает наименьшего значения, равного $\gamma_{1} \gamma_{2}, n р и$

$$
F(x, y)=\sum_{i=0}^{\alpha} b_{i}^{*} \pi_{i}(x) \sum_{k=0}^{\beta} c_{k}^{*} \omega_{k}(y) .
$$

Для доказательства этой леммы достаточно для функции (6а) проверить справедливость условий (6). Отметим, что эта лемма является обобщением теоремы из работы $\left[{ }^{3}\right]$.

Пусть функция $f(x, y) \in \widehat{W}_{q}^{r s}$. Применяя повторно формулу [ $\left.{ }^{4}\right]$ разложения функции одной переменной по многочленам Бернулли, имеем 


$$
\begin{aligned}
f(x, y)= & \int_{0}^{1} \int_{0}^{1} f(t, u) d t d u-\frac{1}{r !} \int_{0}^{1} \int_{0}^{1} f^{(r, 0)}(t, u) B_{r}^{*}(x-t) d t d u- \\
& -\frac{1}{s !} \int_{0}^{1} \int_{0}^{1} f^{(0, s)}(t, u) B_{s}^{*}(y-u) d t d u+ \\
+ & \frac{1}{r ! s !} \int_{0}^{1} \int_{0}^{1} f^{(r, s)}(t, u) B_{r}^{*}(x-t) B_{s}^{*}(y-u) d t d u .
\end{aligned}
$$

В дальнейшем, согласно $\left[{ }^{5}\right]$, будем считать формулы (2) точными для постоянной, т. е.

$$
\sum_{i=1}^{m} \sum_{k=1}^{n} A_{i k 00}=1
$$

Из свойств периодических функций следует, что

$$
\int_{0}^{1} \varphi_{f}^{(r)}(t) d t=\int_{0}^{1} \psi_{f}^{(s)}(u) d u=\int_{0}^{1} f^{(r, s)}(t, u) d u=\int_{0}^{1} f^{(r, s)}(t, u) d t=0 .
$$

Подставляя выражение (7) в формулу (2) и учитывая равенства (8) и (9), получим

$$
\begin{aligned}
R_{m n}(f)= & \frac{1}{r !} \int_{0}^{1} \varphi_{f}^{(r)}(t)\left[K_{1}(t)+C_{1}\right] d t+\frac{1}{s !} \int_{0}^{1} \psi_{f}^{(s)}(u)\left[K_{2}(u)+C_{2}\right] d u- \\
& -\frac{1}{r ! s !} \int_{0}^{1} \int_{0}^{1} f^{(r, s)}(t, u)\left[K_{3}(t, u)+\varphi(t)+\psi(u)\right] d t d u
\end{aligned}
$$

где

$$
\begin{aligned}
& K_{1}(t)=\sum_{i=1}^{m} \sum_{k=1}^{n} \sum_{j=0}^{\rho_{i}} A_{i k j 0} B_{r}^{*(j)}\left(x_{i}-t\right), \\
& K_{2}(u)=\sum_{i=1}^{m} \sum_{k=1}^{n} \sum_{l=0}^{\sigma_{h}} A_{i k 0 l} B_{s}^{*(l)}\left(y_{k}-u\right), \\
& K_{3}(t, u)=\sum_{i=1}^{m} \sum_{k=1}^{n} \sum_{j=0}^{\rho_{i}} \sum_{l=0}^{\sigma_{k}} A_{i k j l} B_{r}^{*(j)}\left(x_{i}-t\right) B_{s}^{*(l)}\left(y_{k}-u\right),
\end{aligned}
$$

$C_{1}, C_{2}$ - произвольные постоянные, $\varphi(t), \psi(u)$ - произвольные функции из $L_{p}(0,1)$. Отсюда с помощью неравенства Гёльдера получаем оценку

$$
\begin{gathered}
\left|R_{m n}(f)\right| \leqslant \frac{M}{r !} \min _{C_{1}}\left\|K_{1}(.)+C_{1}\right\|_{p}+\frac{P}{s !} \min _{C_{2}}\left\|K_{2}(.)+C_{2}\right\|_{p}+ \\
+\frac{Q}{r ! s !} \min _{\varphi, \psi \in L_{p}(0,1)}\left\|K_{3}(., .)+\varphi(.)+\psi(.)\right\|_{p}=\frac{M}{r !}\left\|K_{1}(.)+C_{1}^{*}\right\|_{p}+ \\
+\frac{P}{s !}\left\|K_{2}(.)+C_{2}^{*}\right\|_{p}+\frac{Q}{r ! s !}\left\|K_{3}(., .)+\varphi^{*}(.)+\psi^{*}(.)\right\|_{p .}
\end{gathered}
$$


Из необходимых условий минимума следуют равенства

$$
\begin{aligned}
& \int_{0}^{1}\left|K_{1}(x)+C_{1}^{*}\right|^{p-1} \operatorname{sign}\left(K_{1}(x)+C_{1}^{*}\right) d x= \\
& =\int_{0}^{1}\left|K_{2}(y)+C_{2}^{*}\right|^{p-1} \operatorname{sign}\left(K_{2}(y)+C_{2}^{*}\right) d y=0,
\end{aligned}
$$

$$
\begin{aligned}
& \int_{0}^{1}\left|K_{3}(x, y)+\varphi^{*}(x)+\psi^{*}(y)\right|^{p-1} \operatorname{sign}\left[K_{3}(x, y)+\varphi^{*}(x)+\psi^{*}(y)\right] d x \equiv 0, \\
& \int_{0}^{1}\left|K_{3}(x, y)+\varphi^{*}(x)+\psi^{*}(y)\right|^{p-1} \operatorname{sign}\left[K_{3}(x, y)+\varphi^{*}(x)+\psi^{*}(y)\right] d y \equiv 0 .
\end{aligned}
$$

Так как для принадлежащей в силу этих равенств множеству $\not{W_{q}^{r s}}$ функции

$$
\begin{aligned}
& f_{0}(x, y)=\frac{M\left\|K_{1}(.)+C_{1}^{*}\right\|_{p}^{1-p}}{r !} \int_{0}^{1} B_{r}^{*}(x-t)\left|K_{1}(t)+C_{1}^{*}\right|^{p-1} \operatorname{sign}\left(K_{1}(t)+\right. \\
& \left.+C_{1}^{*}\right) d t+\frac{1}{s !} P\left\|K_{2}(.)+C_{2}^{*}\right\|_{p}^{1-p} \int_{0}^{1} B_{s}^{*}(y-u)\left|K_{2}(u)+C_{2}^{*}\right|^{p-1} \operatorname{sign}\left(K_{2}(u)+\right. \\
& \left.+C_{2}^{*}\right) d u-\frac{1}{r ! s !} Q\left\|K_{3}(., .)+\varphi^{*}(.)+\psi^{*}(.)\right\|{ }_{p}^{1-p} \int_{0}^{1} \int_{0}^{1} B_{r}^{*}(x-t) B_{s}^{*}(y-u) \times \\
& \quad X\left|K_{3}(t, u)+\varphi^{*}(t)+\psi^{*}(u)\right|^{p-1} \operatorname{sign}\left[K_{3}(t, u)+\varphi^{*}(t)+\psi^{*}(u)\right] d t d u
\end{aligned}
$$

неравенство (10) обращается в равенство, то справедливо соотношение $R_{m n}=\frac{M}{r !}\left\|K_{1}(.)+C_{1}^{*}\right\|_{p}+\frac{P}{s !}\left\|K_{2}(.)+C_{2}^{*}\right\|_{p}+\frac{Q}{r ! s !}\left\|K_{3}(., .)+\varphi^{*}(.)+\psi^{*}(.)\right\|_{p}$.

Таким образом, для построения наилучшей на множестве $\mathscr{W}_{q}^{r s}$ кубатурной формулы (2) достаточно минимизировать величину (11).

Обозначим через $\mathscr{W}_{q}^{r}$ множество всех функций $f(x)$, имеющих абсолютно непрерывную производную порядка $r-1$ на всей числовой оси и удовлетворяющих условиям

$$
f(x+1) \equiv f(x), \quad\left\|f^{(r)}(.)\right\|_{q} \leqslant 1 .
$$

Через $C_{i j}^{*}\left(i=1, \ldots, m ; j=0, \ldots, \varrho_{i}\right)$ обозначим веса, а через $\delta_{1} / r$ ! точную оценку ошибки наилучшей $\left[{ }^{1}\right]$ на множестве $\widetilde{W}_{q}^{r}$ формулы

$$
\int_{0}^{1} f(x) d x=\sum_{i=1}^{m} \sum_{j=0}^{\rho_{i}} C_{i j} f^{(j)}\left(x_{i}\right)+R_{m}(f),
$$

где узлы $0 \leqslant x_{1}<\ldots<x_{m} \leqslant 1\left(x_{m}-x_{1} \neq 1\right)$ фиксированы.

Аналогично, через $D_{k l}^{*}\left(k=1, \ldots, n ; l=0, \ldots, \sigma_{k}\right)$ обозначим 
веса, через $\delta_{2} / s$ ! точную оценку ошибки наилучшей на множестве $\tilde{W}_{q}^{s}$ формулы

$$
\int_{0}^{1} f(y) d y=\sum_{k=1}^{n} \sum_{l=0}^{r \sigma_{k}} D_{k l} f^{(l)}\left(y_{k}\right)+R_{n}(f)
$$

с фиксированными узлами $0 \leqslant y_{1}<\ldots<y_{n} \leqslant 1\left(y_{n}-y_{1} \neq 1\right)$.

Т е о е м а 1. Наилучшая на множестве $\widetilde{W}_{q}^{r s}$ формула (2) с фиксированными узлами $\left(x_{i}, y_{k}\right)(i=1, \ldots, m ; k=1, \ldots, n) *$ имеет веса

$$
\begin{gathered}
A_{i k j l}=C_{i j}^{*} D_{k l}^{*} \\
\left(i=1, \ldots, m ; j=0, \ldots, \varrho_{i} ; \quad k=1, \ldots, n ; l=0, \ldots, \sigma_{k}\right)
\end{gathered}
$$

и точную оценку ошибки

$$
R_{m n}=M \frac{\delta_{1}}{r !}+P \frac{\delta_{2}}{s !}+Q \frac{\delta_{1} \delta_{2}}{r ! s !} .
$$

Д ок а з а т л в с т о. Как следует из $\left[{ }^{1,6,7}\right]$, при условиях

$$
\sum_{i=1}^{m} C_{i 0}=1, \quad \sum_{k=1}^{n} D_{k 0}=1
$$

имеют место неравенства

$$
\begin{aligned}
& \int_{0}^{1}\left|\sum_{i=1}^{m} \sum_{j=0}^{\rho_{t}} C_{i j} B_{r}^{*(j)}\left(x_{i}-t\right)+C_{0}\right|^{p} d t \geqslant \delta_{1}^{p}, \\
& \int_{0}^{1}\left|\sum_{k=1}^{n} \sum_{l=0}^{\sigma_{k}} D_{k l} B_{s}^{*(l)}\left(y_{k}-u\right)+D_{0}\right|^{p} d t \geqslant \delta_{2}^{p},
\end{aligned}
$$

которые обращаются в равенства при

$$
\begin{array}{lll}
C_{i j}=C_{i j}^{*} & \left(i=1, \ldots, m ; j=0, \ldots, \varrho_{i}\right), & C_{0}=C_{0}^{*}, \\
D_{k l}=D_{k l}^{*} & \left(k=1, \ldots, n ; \quad l=0, \ldots, \sigma_{k}\right), & D_{0}=D_{0}^{*},
\end{array}
$$

где $C_{0}^{*}, D_{0}^{*}$ - некоторые постоянные.

Отсюда и из леммы 3 следует, что при любых $\varphi(t), \psi(u) \in L_{p}(0,1)$ имеет место неравенство

$$
\int_{0}^{1} \int_{0}^{1}\left|K_{3}(t, u)+\varphi(t)+\psi(u)\right|^{p} d t d u \geqslant \delta_{1}^{p} \delta_{2}^{p},
$$

для обращения которого в равенство необходимо условие (14). Кроме того, из неравенств (15) следуют неравенства

$$
\left.\int_{0}^{1}\left|K_{1}(t)+C_{1}\right|\right|^{p} d t \geqslant \delta_{1}^{p}, \quad \int_{0}^{1}\left|K_{2}(u)+C_{2}\right|^{p} d u \geqslant \delta_{2}^{p},
$$

также обращающиеся в равенства при весах (14) и $C_{1}=C_{0}^{*}, C_{2}=D_{0}^{*}$, что и доказывает теорему.

Обозначим через $\bar{x}_{i}, \bar{C}_{i j}\left(i=1, \ldots, m ; j=0, \ldots, \varrho_{i}\right), \bar{\delta}_{1} / r !$ узлы,

* Формула (2) называетоя наилучшей на $W_{q}^{r s}$ с фиксированными узлами, если ее веса выбраны из условия минимума величины $R_{m n}$ при заданных узлах. 
веса и точную оценку ошибки наилучшей на множестве $\mathbb{W}_{q}^{r}$ формулы $(12)$, а через $\bar{y}_{k}, \bar{D}_{k l}\left(k=1, \ldots, n ; l=0, \ldots, \sigma_{k}\right), \bar{\delta}_{2} / s !-$ узлы, веса и точную оценку ошибки наилучшей на множестве $\widetilde{W}_{q}^{s}$ формулы (13).

Т е о рем а 2. Наилучшая на множестве $\widetilde{W}_{q}^{r s}$ формула (2) имеет узльь

$$
\left(x_{i}, y_{k}\right)=\left(\bar{x}_{i}, \bar{y}_{k}\right) \quad(i=1, \ldots, m ; k=1, \ldots, n),
$$

веса

$$
\begin{gathered}
A_{i k j l}=\bar{C}_{i j} \bar{D}_{k l} \\
\left(i=1, \ldots, m ; j=0, \ldots, \varrho_{i} ; k=1, \ldots, n ; l=0, \ldots, \sigma_{k}\right)
\end{gathered}
$$

и точную оценку ошибки

$$
R_{m n}=M \frac{\bar{\delta}_{1}}{r !}+P \frac{\bar{\delta}_{2}}{s !}+Q \frac{\bar{\delta}_{1} \bar{\delta}_{2}}{r ! s !}
$$

Для доказатель с в а этой теоремы следует заметить, что величина $\delta_{1}$ как функция от $x_{1}, \ldots, x_{m}$ достигает наименьшего значения, равного $\bar{\delta}_{1}$, при $x_{i}=\bar{x}_{i}(i=1, \ldots, m)$, а величина $\delta_{2}$ как функция от $y_{1}, \ldots, y_{n}$ достигает наименьшего значения, равного $\bar{\delta}_{2}$, при $y_{k}=\bar{y}_{k}$ $(k=1, \ldots, n)$, а затем воспользоваться результатом теоремы 1 .

Одним из возможных применений полученных результатов является следующая

Т ео рем а 3. Наилучшая на множестве $\widetilde{W}_{q}^{r s}$ формула

$$
\int_{0}^{1} \int_{0}^{1} f(x, y) d x d y=\sum_{i=1}^{m} \sum_{k=1}^{n} A_{i k} f\left(x_{i}, y_{k}\right)+R_{m n}(f)
$$

имеет узльь

$$
x_{i}=\frac{i-\theta_{1}}{m}, \quad y_{k}=\frac{k-\theta_{2}}{n} \quad(i=1, \ldots, m ; k=1, \ldots, n),
$$

где $\theta_{1} u \theta_{2}$ произвольные числа, удовлетворяющие условию

$$
0 \leqslant \theta_{1} \leqslant 1 / m, \quad 0 \leqslant \theta_{2} \leqslant 1 / n,
$$

веса

$$
A_{i k}=1 / m n \quad(i=1, \ldots, m ; k=1, \ldots, n)
$$

и точную оценку ошибки

$$
R_{m n}=\frac{M}{m^{r}} B_{r, p}+\frac{P}{n^{s}} B_{s, p}+\frac{Q}{m^{r} n^{s}} B_{r, p} B_{s, p},
$$

где

$$
B_{j, p}=\frac{1}{j !} \min _{c}\left\|B_{j}(\cdot)-c\right\|_{p} .
$$

Этот результат непосредственно вытекает из теоремы 2 и работы $\left[{ }^{8}\right]$.

Полученные результаты без труда обобщаются на случай функций произвольного числа переменных, а также имеют место и при $q=1, \infty$, 


\section{ЛИТЕ РАТ У РА}

1. Никольский С. М., Квадратурные формулы, М., 1974.

2. Соболев С. Л., Введение в теорию кубатурных формул, М., 1974.

3. Л е вин М., Изв. АН ЭССР, Физ. Матем., 18, 464 (1969).

4. К рылов В. И., Приближенное вычисление интепралов, М., 1967.

5. Л е в и н М., Изв. АН ЭССР, Физ. Матем., 20, 90 (1971).

6. Л евин М. И., Гиршович Ю. М., Арро В. К., Докл. АН СССР, № 226, 51 (1976).

7. Ги р шов ич Ю. М., Л ев ин М. И., Тр. Таллинск. политехн. ин-та, 393, 21 (1976).

8. Женсы к б ае в А. А., Докл. АН СССР, № 227, 227 (1976).

таллинский политехнический институт
Поступила в редакцию 30/IV 1976

\section{LEVIN, J. GIRSOVITS}

\section{PERIOODILISTE FUNKTSIOONIDE HULKADE PARIMAD KUBATUURVALEMID}

Funktsioonihulkadele $W_{q}^{\text {rs }}$, mis rahuldavad tingimust (1), on leitud parim valem (2), mille erijuht (16) omab sởlmi (17), kaale (18) ja veahinnangut (19).

\section{LEVIN, Y. GIRSHOVICH}

\section{OPTIMAL CUBATURE FORMULAE FOR SETS OF PERIODICAL FUNCTIONS}

The optimal cubature formula (2) for the sets $\mathscr{W}_{q}^{r s}$ of functions satisfying (1) is constructed. The optimal formula (16) has knots (17), coefficients (18) and exact error estimation (19). 